\title{
Conférence - Analyse systémique des distorsions de la communication entre l'hôpital et ses usagers
}

\section{Françoise Rougeul}

\section{(2) OpenEdition}

12 Journals

Édition électronique

URL : http://journals.openedition.org/communicationorganisation/3004

DOI : 10.4000/communicationorganisation.3004

ISSN : $1775-3546$

Éditeur

Presses universitaires de Bordeaux

Édition imprimée

Date de publication : 1 mai 1994

ISSN : 1168-5549

Référence électronique

Françoise Rougeul, «Conférence - Analyse systémique des distorsions de la communication entre I'hôpital et ses usagers ", Communication et organisation [En ligne], HS N 1 | 1994, mis en ligne le 27 mars 2012, consulté le 19 avril 2019. URL : http://journals.openedition.org/

communicationorganisation/3004; DOI : 10.4000/communicationorganisation.3004

Ce document a été généré automatiquement le 19 avril 2019

(c) Presses universitaires de Bordeaux 


\title{
Conférence - Analyse systémique des distorsions de la communication entre l'hôpital et ses usagers
}

\author{
Françoise Rougeul
}

1 L'analyse systémique est une approche originale qui permet de comprendre autrement les difficultés de la communication.

2 Nous prendrons comme exemple, l'histoire d'une naissance pourrait-on dire «miraculeuse » mais pour commencer, il nous faut définir brièvement quelques concepts propres à ce type d'analyse.

\section{Système}

3 On entend par système un ensemble d'éléments reliés par des interactions soit trop puissantes soit trop complexes pour qu'on puisse considérer un élément isolément. Dans le cas des systèmes vivants, on note l'émergence d'une autre propriété, l'organisation en fonction d'un but, ce qui peut permettre de distinguer des sous-systèmes à l'intérieur d'un même grand système. Ainsi, au sein du système hôpital, pourra-t-on définir plusieurs sous-systèmes. Dans le cas qui nous occupe, il y aura principalement le soussystème soigné (le couple) et le sous-système soignant.

\section{Autorégulation}

4 Les systèmes humains sont des systèmes vivants qui évoluent en passant par différentes phases. Ces systèmes sont autorégulés ce qui leur permet de garder identité et cohésion au cours du temps tout en changeant de fonctionnement lorsque celui-ci n'est plus adapté. Cet équilibre délicat entre permanence et changement s'opère grâce à des mécanismes de feed-back positifs et négatifs. Les premiers poussent à la conservation du 
fonctionnement avec un risque d'immobilisme ou même de blocage, les seconds au changement mais avec des risques d'emballement.

\section{Communication}

5 Dans les systèmes humains, les interactions sont constituées par des communications or la communication est un processus complexe. La complexité tient au fait que les boucles de rétroaction enchevêtrent les messages à l'intérieur d'un même niveau où ils s'autorégulent par les mécanismes de feed-back déjà décrits. Mais elle comprend également des niveaux différents et les boucles de rétroaction vont s'étendre entre les niveaux.

Or ces niveaux sont hiérarchisés, par ordre d'importance, on distingue :

7 - le niveau informatif, en général véhiculé par le langage verbal ou écrit. Dans notre cas, une jeune femme dira « je suis enceinte ».

8 - le niveau relationnel traduit la relation entre les partenaires ou sous-systèmes engagés dans un processus de communication. Ce niveau passe plutôt par le comportement. Il est considéré d'un niveau supérieur au précédent car il indique comment le premier message doit être compris et interprété.

9 - enfin le niveau contextuel représente le système de valeurs et d'usages qui régissent les pensées, désirs et comportements des membres de chaque sous-système. Ce niveau dit mythique est fondamental car il détermine les deux premiers ainsi que nous allons le voir dans notre exemple clinique.

\section{Histoire d'une naissance miraculeuse}

Il s'agit d'un jeune couple dont l'histoire est racontée par J. PLUYMAKERS dans son livre « Famille, institution et approche systémique ».

11 Ce couple qui attend son premier enfant, décide de choisir un gynécologue qui serait «non un simple technicien mais un accompagnateur prêt à partager avec eux toute l'aventure, de la préparation jusqu'à l'accouchement compris »... Cela les amènera à prendre par téléphone plusieurs rendez-vous, qu'ils commenceront de la même manière «dans un premier temps, nous venons simplement pour faire connaissance et c'est seulement après que nous déciderons comment et avec qui, nous préparerons cette naissance. »

12 Face à cette demande inhabituelle, les médecins consultés présenteront tous le même comportement, "comme s'ils s'étaient donnés le mot.». Ils constitueront un dossier, prescriront des examens complémentaires et proposeront un autre rendez-vous, chacun des praticiens se comportant comme s'il était « l'élu. »

De même par rapport au père, ils adopteront la même attitude, chaque fois que le père posera une question, c'est à la mère que la gynécologue répondra ou tout au moins, c'est elle qu'il fixera des yeux. Pour finir, le choix du couple se portera sur le gynécologue avec lequel les rapports sont le plus personnalisés et sur une clinique type LEBOYER qui leur semble « le moins hospitalier et le plus accueillant de tous les établissements».

Le jour de l'accouchement est marqué par une incroyable succession de mésaventures : 
15 - le nombre d'accouchements est exceptionnel, le personnel fourbu. Toutes les chambres et salles étant occupées, ils aboutissent à l'étage du dessous, en chirurgie.

16 - ils attendent des heures. L'appareil qui contrôle les battements du cœur de la mère et de l'enfant semble impossible à régler, puis il fonctionne, enfin il commence à sonner mettant tout l'étage en alerte...Le drame commence, raconté par le père «j'assistais, statue muette par obligation, à un étonnant ballet. C'était l'infirmière puis le médecin qui venaient se pencher sur les cadrans avant de discuter à mi-voix avec des regards qui me transperçaient comme si j'étais l'homme invisible. Il va falloir se décider disait-on » et puis encore « ça ne va pas bien, hein? » ou " pratiquer la césarienne? ?.

17 «J'aurais aimé qu'on tienne compte de nous les parents, qu'on me demande ne fut-ce qu'un acquiescement à propos d'une décision qui était en train de se prendre à $2 \mathrm{mn}$ de moi. On rallume tout l'éclairage, adieu les lumières tamisées de LEBOYER... C'est la salle d'opération en urgence. Pour la mère, les contractions douloureuses continuant, elle crie. «Taisez-vous !... » hurlent les infirmières, vertueusement indignées, une future opérée, ça ne crie pas... On m'avait affublé d'une blouse verte et j'étais là, spectateur privilégié d'un formidable décalage. "

18 Cela dit, la césarienne se passe bien, une aspiration des mucosités pouvant gêner l'enfant est pratiquée avec le matériel de réanimation descendu du service maternité, l'atmosphère se détend, on laisse même le père donner un bain à l'enfant, puis tout le monde remonte dans la chambre.

Quelque temps après, le bébé bleuit, la grand mère arrivée essaie de le faire respirer, on sonne l'infirmière qui ne répond pas. Le père court à sa recherche, ne la trouve nulle part, redescend en chirurgie. Il tombe sur une infirmière de chirurgie qui, ne trouvant pas non plus sa collègue, décide d'appeler les urgences. Deux médecins arrivent mais leur matériel de réanimation n'est pas adapté aux nouveau-nés... Ils ne savent pas où est rangé le matériel de la maternité... Lorsque le père qui était présent à l'opération leur indiquera où il a été mis, ils ne sauront pas le faire fonctionner et c'est également lui qui, ayant assisté le matin à l'aspiration des mucosités, suggérera un geste qui permettra aux médecins de mettre en marche l'appareil...

20 L'infirmière de maternité a été retrouvée, elle était dans une autre chambre et elle avait oublié de brancher la sonnette d'appel... Les médecins lui conseillent d'appeler un pédiatre. La décision est prise de placer l'enfant en couveuse, l'enfant respire, le père aussi, pensant que l'enfant sera mieux surveillé que dans une chambre d'accouchée.

21 Pas du tout, dans la salle des couveuses, il n'y a qu'un autre bébé. L'infirmière place l'enfant dans une couveuse qui disjoncte à plusieurs reprises puis se remet en marche sans qu'on sache pourquoi... Elle pousse le bout du tuyau à oxygène par une petite fenêtre, le bout du tuyau se retrouvant près du nez et des yeux du bébé... Le père regarde très étonné. Resté seul, il va scruter la couveuse sous tous les angles et réagir par rapport à ce qui lui apparaît illogique, c'est-à-dire qu'il ouvrira régulièrement la couveuse pour renouveler l'air et s'assurer qu'il n'y a pas trop d'oxygène. Il passera ainsi toute la nuit dans l'angoisse.

Dès que l'infirmière de jour arrive, elle tombe en arrêt devant la couveuse, arrache le tuyau et sans rien dire le rebranche là où il fallait. Suit une explication générale avec le médecin-chef au cours de laquelle le père tout en soulignant la gravité de ce qui s'est passé et en faisant des réserves par rapport à d'éventuelles séquelles, annoncera son 
intention «de ne pas leur faire d'histoires» car, pour lui, il était clair que les circonstances avaient trop exigé de l'infirmière.

La fin racontée par le père, ne manque pas d'intérêt «si, dans le service, on nous a couvert d'attentions, presque cajolés, en revanche nous nous heurtâmes à l'hostilité des services administratifs... À cause de nos mésaventures, nous avions oublié de payer l'acompte... or «il n'est pas dans l'habitude de la maison de ne pas en respecter les règles... Bref, on nous fit la leçon !...»

\section{Analyse systémique}

24 Pour ne pas trop compliquer l'analyse, nous mettrons de coté le système administratif qui n'est pas directement impliqué dans notre histoire, et nous limiterons aux deux soussystèmes : «le jeune couple » et « les soignants ». Ces deux sous-systèmes sont organisés en fonction d'un but, la survie mais la crise fait apparaitre que la survie n'a pas le même sens pour l'un et pour l'autre. Pour le jeune couple la survie implique que son premier enfant naisse normalement et conformément à leurs vœux, en ce qui concerne la clinique, la survie implique aussi qu'il n'y ait ni accident ni histoire susceptible d'entamer sa réputation. Les sous-systèmes sont donc différents, leur contexte différent et l'essentiel de cette histoire peut être décrite comme un conflit de contextes.

\section{Contexte hospitalier :} l'erreur. En cas d'accident, il va être tenu pour responsable ce qui peut ternir sa réputation voire entraîner sa perte sous forme d'une perte de clientèle quand il s'agit d'une clinique. Or les soignants sont des êtres humains et comme tels faillibles. Pour se préserver quelque peu de l'humaine faiblesse, il faut s'abriter derrière une technique sans faille, édicter des règles qui tendront à devenir de plus en plus rigides. 

difficulté de l'humanité d'une relation qui repose sur un déni.....

\section{Contexte du couple}

Nous ne le connaissons que d'après cette courte histoire où ils sont présentés comme choisissant de vivre l'arrivée de leur premier enfant comme une aventure où ils auront besoin d'un «accompagnateur». De plus, ils sont déterminés à vérifier, avant de s'engager avec un praticien, si cette personne est digne de l'honneur qui lui est fait...

3 Ce contexte qui évoque celui de futurs explorateurs à la recherche d'un guide est complètement différent du contexte médical auquel le terme d'aventure est inconnu, en outre c'est le couple qui se met en position haute...

Voyons maintenant comment ces différents éléments vont jouer dans la dynamique de notre histoire...

Dès la première consultation, les contextes s'opposent.

Les médecins consultés ne peuvent s'adapter à une demande trop inhabituelle pour n'être pas provocante. La seule réponse possible, est de n'en pas tenir compte. Dès lors, bien que le jeune couple se soit clairement exprimé au téléphone, sa demande est ramenée à l'intérieur du cadre prévu. À partir des mots-clés, «je suis enceinte », le rituel se met automatiquement en place, le praticien ouvre un dossier, examine la patiente, prescrit des examens et donne un prochain rendez-vous, « tout comme s'il était l'élu ».

Quelque chose de semblable se joue dans la place du futur père. D'un côté, l'idéologie du moment la juge strictement nécessaire, c'est en couple que doit se préparer une naissance. La pratique privée qui est celle des gynécologues et des cliniques doit tenir compte de ce contexte socioculturel, eu égard à leur clientèle. D'un autre côté, les règles beaucoup plus anciennes qui découlent du contexte médical et s'actualisent dans sa pratique, n'ont guère changé. Il se crée donc une situation assez étonnante pour le futur père, qui invité chaleureusement en fonction de l'idéologie du moment, « est là sans être là », puisque chaque fois qu'il s'exprime, c'est à la mère qu'on répond.... «très étrange, écrit-il, de se sentir comme père tout juste toléré

Le père fait alors l'expérience de la contradiction entre les différents niveaux de la communication, contextuel, verbal et comportemental, le comportement du gynécologue qui reste en accord avec le contexte médical, lui renvoyant implicitement le message relationnel « tu es là sans être là ».

39 Cette rigidité propre au contexte médical va se retrouver amplifiée lors du séjour à la clinique. Rappelons comme élément fondamental de ce contexte la règle selon laquelle l'institution est supposée savoir mieux que le patient ce que le patient doit subir. Or que se passe-t-il si le système hospitalier entre en crise, c'est-à-dire s'il devient probable qu'il ne puisse remplir cette fonction? Quelles sont ses capacités d'autorégulation?

Dans notre cas, on commence par observer un mécanisme de feed-back négatif puissant. Les règles de la pratique médicale anciennes sont réactualisées. Ainsi que le décrit le père, affublé de sa blouse verte dans la salle d'opération «j'étais là, spectateur privilégié d'un formidable décalage...» (adieu les lumières tamisées type LE BOYER, décision d'intervention prise en dehors du couple, la femme qui se fait disputer parce qu'elle crie....) 
Par la suite, les mécanismes de feed-back positifs prennent le relais, mais le système risque l'emballement ainsi que le montrera l'incident de la couveuse.

L'infirmière de la maternité restée introuvable, entraîne l'intervention de nombreuses personnes qui, toutes vont devoir réagir, hors des règles habituelles... sans savoir si cela va ramener les choses à la normale... ou créer de nouveaux problèmes...

L'infirmière de chirurgie quitte son service sans prévenir... Les médecins des urgences se trouvent perdus dans la maternité de leur hôpital et «le père se retrouve en première ligne sur un terrain inconnu

En fait, l'acmé de la crise se situe au moment de l'absence de l'infirmière de la maternité. Le système en sortira par une alliance étonnante qui va émerger, à partir d'un accord implicite de chacun sur la défaillance du système-hôpital. Cela se noue au moment où le père déclare savoir où se trouve l'appareil de réanimation pour les nouveau nés. On peut d'ailleurs se demander si la cause réelle de celle alliance est la reconnaissance du dysfonctionnement institutionnel ou la présence d'un étranger-témoin en la personne du père...

5 Toujours est-il que, dès lors, contextuellement, le père est désigné comme co-thérapeute. Il va contribuer non seulement à sauver son enfant mais encore à protéger la réputation de l'hôpital, au moment où le fonctionnement de celui-ci est gravement défaillant.

Mais cela pointe les risques immenses que le personnel fait courir à l'institution-hôpital, dès le moment où il accepte que les règles habituelles soient bouleversées. En effet, après les incidents de la réanimation, il n'est pas question de demander au père de retourner dans sa chambre et lui... n'est pas près de renoncer à son contrôle.

Ainsi peut-on relire l'histoire de la couveuse. La présence du père amplifie la disqualification de l'infirmière, encore sous le choc de son « absence ». Plus le père est là, moins l'infirmière peut se montrer compétente.... C'est à ce moment que le système risque un emballement qui se traduit par un jeu de non-dits insensés... L'infirmière ne peut s'expliquer devant cette couveuse qui refuse de se mettre en marche comme il faudrait. Le père regarde et se tait. Il n'ose pas intervenir.... devant l'infirmière. C'est après, qu'il adoptera, seul... un comportement régulateur mais qui aurait pu être inadéquat.

Un des intérêts de cette analyse, c'est de montrer que le fonctionnement de cette institution est peut-être moins critiquable qu'on serait tenté de le croire au premier abord. En effet, elle a été capable de s'adapter...la place laissée au père, a permis à des règles rigides de gagner en flexibilité, même si cette place a émergé des circonstances... On peut prévoir le drame qui aurait pu se passer si une rigidité institutionnelle plus grande avait empêché toute intrusion extérieure en son sein...

\title{
Évolution des difficultés de la communication en milieu hospitalier
}

\author{
Ces difficultés nous paraissent en rapport avec l'évolution des contextes. \\ Les soignés deviennent des « clients ». \\ De ce fait, ils sont décrits comme de plus en plus « exigeants ». Mieux au courant de leurs \\ droits, ils ont tendance à les faire valoir : droit à l'information, droit de refuser certains \\ traitements ou droit d'attaquer en cas de faute médicale. Rappelons que si les médecins
}


n'ont pas droit à l'erreur, ils en commettent quand même... et les patients qui auparavant réagissaient comme des moutons, deviennent eux ou leurs familles plus facilement « enragés »... analyse systémique des comportements des soignants. Ils ont ce comportement à ce moment par rapport à cet enfant, mais que s'est-il passé dans le service pendant le même temps? En effet, on a toujours l'impression qu'on ne soigne qu'un malade à la fois, mais l'infirmière était peut-être occupée avec une autre urgence...

Qu'engendrera donc ce nouveau contexte qui évolue vers un accroissement du professionnalisme, de l'efficacité technique et financière ainsi que du clientélisme?

On pourrait supposer que les anciens "patients » seront davantage considérés dans la mesure où ils deviennent des « clients ».

À moins que l'équipe hospitalière ne réagisse à ce contexte de stress accru par un mécanisme de feed-back négatif puissant ainsi que nous l'avons vu dans notre exemple clinique. Cela consisterait à donner le moins de prise possible à d'éventuelles attaques des patients, en s'abritant derrière une technicité sinon irréprochable du moins incompréhensible aux non spécialistes et en dépersonnalisant les rapports au maximum.

\section{Questions}

Thérèse PSUIK : Je me demande s'il ne faudrait pas recouper votre analyse avec une Françoise ROUGEUL : J'ai peut-être parlé un peu vite. C'est vrai que le premier contact avec la salle d'accouchement s'est fait dans un moment de crise. Il est vrai aussi que, quand la crise est passée, ils ont été «cajolés ». Il faut dire qu'il s'en sont bien sortis et que tout le monde a eu de la chance, y compris et surtout l'enfant. 
65 Georgette POLI : À propos des problèmes d'accouchement, il est vrai qu'on a moins voulu médicaliser ceux-ci. Si nous médicalisons trop, on nous accuse d'être trop rigides et impersonnels. Il est vrai que ce genre d'équipe est rigide, mais compétente et il faudrait essayer d'équilibrer un peu les deux. D'autre part, une chose m'a choquée. Vous avez parlé d'aventure à propos de ce jeune couple, et d'un gynécologue qui voulait partager l'aventure avec lui. Je crois que l'aventure est au sein du couple. Pourquoi faut-il chercher systématiquement une aventure spirituelle ou affective avec un médecin?

Françoise ROUGEUL : C'est ce qui fait partie, de l'originalité de leur contexte, à savoir qu'ils posent une demande qui est absolument inhabituelle pour un gynécologue. Ce qui explique que, très normalement les gynécologues n'en tiennent pas compte. Dans cette approche, je n'accuse personne. Je propose là une lecture qui permet de comprendre pourquoi les gens ne se comprennent pas, parce que chaque fois le père s'étonne en se disant : "Comment ça se fait, il ne me regarde pas, il regarde ma femme » et c'est vrai qu'ils mettent les médecins dans une position abominable... et leur contexte était en opposition complète avec le contexte médical.

\section{AUTEUR}

FRANÇOISE ROUGEUL

Professeur de Psychopathologie clinique. Laboratoire C.I.R.A.C.C., Université de NiceSophia Antipolis 